УДК 515.12

T. BANAKH, A. RAVSKY

\title{
THE REGULARITY OF QUOTIENT PARATOPOLOGICAL GROUPS
}

\begin{abstract}
T. Banakh, A. Ravsky. The regularity of quotient paratopological groups, Mat. Stud. 49 (2018), 144-149.

Let $H$ be a closed subgroup of a regular Abelian paratopological group $G$. The group reflexion $G^{b}$ of $G$ is the group $G$ endowed with the strongest group topology, which is weaker than the original topology of $G$. We show that the quotient $G / H$ is Hausdorff (and regular) if $H$ is closed (and locally compact) in $G^{b}$. On the other hand, we construct an example of a regular abelian paratopological group $G$ containing a closed discrete subgroup $H$ such that the quotient $G / H$ is Hausdorff but not regular.
\end{abstract}

In this paper we study the properties of the quotients of paratopological groups by their normal subgroups.

By a paratopological group $G$ we understand a group $G$ endowed with a topology $\tau$ making the group operation continuous, see [8]. If, in addition, the operation of taking inverse is continuous, then the paratopological group $(G, \tau)$ is a topological group. A standard example of a paratopological group failing to be a topological group is the Sorgefrey line $\mathbb{L}$, that is the real line $\mathbb{R}$ endowed with the Sorgefrey topology (generated by the base consisting of half-intervals $[a, b), a<b)$.

Let $(G, \tau)$ be a paratopological group and $H \subset G$ be a closed normal subgroup of $G$. Then the quotient group $G / H$ endowed with the quotient topology is a paratopological group, see [3]. Like in the case of topological groups, the quotient homomorphism $\pi: G \rightarrow G / H$ is open. If the subgroup $H \subset G$ is compact, then the quotient $G / H$ is Hausdorff (and regular) provided so is the group $G$, see [3]. The compactness of $H$ in this result cannot be replaced by the local compactness as the following simple example shows.

Example 1. The subgroup $H=\{(-x, x): x \in \mathbb{Q}\}$ is closed and discrete in the square $G=\mathbb{L}^{2}$ of the Sorgenfrey line $\mathbb{L}$. Nonetheless, the quotient group $G / H$ fails to be Hausdorff: for any irrational $x$ the coset $(-x, x)+H$ cannot be separated from zero $(0,0)+H$.

A necessary and sufficient condition for the quotient $G / H$ to be Hausdorff is the closedness of $H$ in the topology of group reflexion $G^{b}$ of $G$.

By the group reflexion $G^{b}=\left(G, \tau^{b}\right)$ of a paratopological group $(G, \tau)$ we understand the group $G$ endowed with the strongest topology $\tau^{b} \subset \tau$ turning $G$ into a topological group. This topology admits a categorial description: $\tau^{b}$ is a unique topology on $G$ such that

- $\left(G, \tau^{b}\right)$ is a topological group;

2010 Mathematics Subject Classification: 22A15, 54H10, 54H11.

Keywords: paratopological group; quotient paratopological group; group reflexion; regularity. doi:10.15330/ms.49.2.144-149

(C) T. Banakh, A. Ravsky, 2018 
- the identity homomorphism id: $(G, \tau) \rightarrow\left(G, \tau^{b}\right)$ is continuous;

- for each continuous group homomorphism $h: G \rightarrow H$ into a topological group $H$ the homomorphism $h \circ \mathrm{id}^{-1}: G^{b} \rightarrow H$ is continuous.

Observe that the group reflexion of the Sorgenfrey line $\mathbb{L}$ is the usual real line $\mathbb{R}$.

For so-called 2-oscillating paratopological groups $(G, \tau)$ the topology $\tau^{b}$ admits a very simple description: its base at the origin $e$ of $G$ consists of the sets $U U^{-1}$, where $U$ runs over open neighborhoods of $e$ in $G$. Following [1] we define a paratopological group $G$ to be 2-oscillating if for each neighborhood $U \subset G$ of the origin $e$ there is another neighborhood $V \subset G$ of $e$ such that $V^{-1} V \subset U U^{-1}$. The class of 2-oscillating paratopological groups is quite wide: it contains all Abelian (more generally all nilpotent) paratopological groups and all saturated paratopological groups. Following I.Guran we define a paratopological group to be saturated if for each neighborhood $U$ of the origin in $G$ its inverse $U^{-1}$ has non-empty interior in $G$.

Given a subset $A$ of a paratopological group $(G, \tau)$ we can talk of its properties in the topology $\tau^{b}$. In particular, we shall say that a subset $A \subset G$ is b-closed in $G$ if it is closed in the topology $\tau^{b}$. Also with help of the group reflexion many useful properties of paratopological groups can be defined.

A paratopological group $G$ is called

- b-separated if the topology $\tau^{b}$ is Hausdorff;

- b-regular if it has a neighborhood base at the origin, consisting of b-closed sets;

- b-compact if $G^{b}$ is compact.

It is clear that each b-separated (and b-regular) paratopological group is functionally Hausdorff (and regular). Conversely, each Hausdorff (resp. regular) 2-oscillating group is b-separated (resp. b-regular), see [1]. On the other hand, there are examples of (nonabelian) Hausdorff paratopological groups $G$ which are not b-separated, see [3], [1]. The simplest example of a b-compact non-compact paratopological group is the Sorgefrey circle $\{z \in$ $\mathbb{C}:|z|=1\}$ endowed with the topology generated by the base consisting of "half-intervals" $\left\{e^{i \varphi}: \varphi \in[a, b)\right\}, a<b$.

Now we are able to state our principal positive result.

Theorem A. Let $H$ be a normal subgroup of a b-separated paratopological group $G$. Then the quotient paratopological group $G / H$ is

1. b-separated if and only if $H$ is closed in $G^{b}$;

2. b-regular if $G$ is b-regular and the set $H$ is locally compact in $G^{b}$.

Proof. Let $\pi: G \rightarrow G / H$ denote the quotient homomorphism.

1. If $H$ is closed in $G^{b}$ then $G^{b} / H$ is Hausdorff as a quotient of a Hausdorff topological group $G^{b}$. Since the identity homomorphism $G / H \rightarrow G^{b} / H$ is continuous, the paratopological group $G / H$ is b-separated.

Now assume conversely that the paratopological group $G / H$ is b-separated. Since the quotient map $\pi^{b}: G^{b} \rightarrow(G / H)^{b}$ is continuous, its kernel $H$ is closed in $G^{b}$.

2. Assume that $G$ is b-regular and $H$ is locally compact in $G^{b}$. It follows that $H$ is closed in $G^{b}$ (this so because the subgroup $H \subset G^{b}$, being locally compact, is complete). Then there is a closed neighborhood $W_{1} \subset G^{b}$ of the neutral element $e$ such that the intersection $W_{1} \cap H$ is compact in $G^{b}$. Take any closed neighborhood $W_{2} \subset G^{b}$ of $e$ such that $W_{2}^{-1} W_{2} \subset W_{1}$. We 
claim that $W_{2} \cap g H$ is compact for each $g \in G$. This is trivial if $W_{2} \cap g H$ is empty. If not, then $g h=w$ for some $h \in H$ and $w \in W_{2}$. Hence $W_{2} \cap g H \subset W_{2} \cap w h^{-1} H=W_{2} \cap w H=$ $w\left(w^{-1} W_{2} \cap H\right) \subset w\left(W_{2}^{-1} W_{2} \cap H\right) \subset w\left(W_{1} \cap H\right)$ and the closed subset $W_{2} \cap g H$ of $G$ lies in the compact subset $w\left(W_{1} \cap H\right)$ of $G$. Consequently, $W_{2} \cap g H$ is compact for any $y \in G$. Let $W_{3} \subset G^{b}$ be a neighborhood of $e$ such that $W_{3}^{-1} W_{3} \subset W_{2}$.

To prove the b-regularity of the quotient group $G / H$, given any neighborhood $U \subset G$ of $e$ it suffices to find a neighborhood $V \subset U$ of $e$ such that $\pi(V)$ is b-closed in $G / H$. By the b-regularity of $G$, we can find a b-closed neighborhood $V \subset U \cap W_{3}$. We claim that $\pi(V)$ is b-closed in $G / H$. Since the identity map $(G / H)^{b} \rightarrow G^{b} / H$ is continuous, it suffices to verify that $\pi(V)$ is closed in the topological group $G^{b} / H$.

Take any point $g H \notin \pi(V)$ of $G^{\mathrm{b}} / H$. It follows from $g H \cap V=\varnothing$ and the compactness of the set $W_{2} \cap g H$ that there is an open neighborhood $W_{4} \subset W_{3}$ of $e$ in $G^{b}$ such that $W_{4}\left(W_{2} \cap g H\right) \cap V=\varnothing$. We claim that $W_{4} z \cap V=\varnothing$ for any $z \in g H$. Assuming the converse, find a point $v \in W_{4} z \cap V$. It follows that $z \notin W_{2}$. On the other hand, $z \in W_{4}^{-1} v \subset W_{4}^{-1} V \subset$ $W_{2}$. This contradiction shows that $W_{4} g H \cap V=\varnothing$ and thus $\pi\left(W_{4} g\right)$ is a neighborhood of $g H$ in $G^{b} / H$, disjoint with $\pi(V)$.

Corollary 1. If $H$ is a b-compact normal subgroup of a b-regular paratopological group $G$, then the quotient paratopological group $G / H$ is b-regular.

Proof. It follows that the identity inclusion $H^{b} \rightarrow G^{b}$ is continuous and thus $H$ is compact in $G^{b}$. Applying the preceding theorem, we conclude that the quotient group $G / H$ is b-regular.

Remark 1. It is interesting to compare the Corollary 1 with a result of Ravsky [3] asserting that the quotient $G / H$ of a Hausdorff (regular) paratopological group $G$ by a compact normal subgroup $H \subset G$ is Hausdorff (regular).

Since for any 2-oscillating paratopological group $G$ the Hausdorff property (the regularity) of $G$ is equivalent to the b-separatedness (the b-regularity), Theorem A implies

Corollary 2. Let $H$ be a normal subgroup of a Hausdorff 2-oscillating paratopological group $G$. Then the quotient paratopological group $G / H$ is

1. Hausdorff if $H$ is closed in $G^{b}$;

2. regular if $G$ is regular and the set $H$ is locally compact in $G^{b}$.

Example 1 supplies us with a locally compact closed subgroup $H$ of a b-regular paratopological group $G=\mathbb{L}^{2}$ such that the quotient $G / H$ is not Hausdorff. Next, we construct a b-regular abelian paratopological group $G$ containing a locally compact b-closed subgroup $H$ such that the quotient is Hausdorff but not regular. This will show that in Theorem A and Corollary 2 the local compactness of $H$ in $G^{b}$ cannot be replaced by the local compactness plus b-closedness of $H$ in $G$.

Our construction is based on the notion of a cone topology (see the respective section of the paper [6] by the second author). Let $G$ be a topological group and $S \subset G$ be a closed subsemigroup of $G$, containing the neutral element $e \in G$. The cone topology $\tau_{S}$ on $G$ consists of sets $U \subset G$ such that for each $x \in U$ there is an open neighborhood $W \subset G$ of $e$ such that $x(W \cap S) \subset U$. It is clear that the group $G$ endowed with the cone topology $\tau_{S}$ is a regular paratopological groups and its neighborhood base at $e$ consists of the sets $W \cap S$, where $W$ 
is a neighborhood of $e$ in $G$. Moreover, the paratopological group $\left(G, \tau_{S}\right)$ is saturated if $e$ is a cluster point of the interior of $S$ in $G$. In the latter case the paratopological group $\left(G, \tau_{S}\right)$ is 2-oscillating and thus b-regular, see [1, Theorem 3].

In the following example using the cone topology we construct a saturated regular paratopological group $G$ containing a b-closed discrete subgroup $H$ with non-regular quotient $G / H$.

Example 2. Consider the group $\mathbb{Q}^{3}$ endowed with the usual (Euclidean) topology. A subsemigroup $S$ of $\mathbb{Q}^{3}$ is called a cone in $\mathbb{Q}^{3}$ if $q \cdot \vec{x} \in S$ for any non-negative $q \in \mathbb{Q}$ and any vector $\vec{x} \in S$.

Fix a sequence $\left(z_{n}\right)$ of rational numbers such that $0<\sqrt{2}-z_{n}<2^{-n}$ for all $n$ and let $S \subset \mathbb{Q}^{3}$ be the smallest closed cone containing the vectors $(1,0,0)$ and $\left(\frac{1}{n}, 1, z_{n}\right)$ for all $n$. Let $\tau_{S}$ be the cone topology on the group $\mathbb{Q}^{3}$ determined by $S$. Since the origin of $\mathbb{Q}^{3}$ is a cluster point of the interior of $S$, the paratopological group $G=\left(\mathbb{Q}^{3}, \tau_{S}\right)$ is saturated and b-regular. Moreover, its group reflexion coincides with $\mathbb{Q}^{3}$.

Now consider the b-closed subgroup $H=\{(0,0, q): q \in \mathbb{Q}\}$ of the group $G$. Since $H \cap S=$ $\{(0,0,0)\}$, the subgroup $H$ is discrete (and thus locally compact) in $G$. On the other hand, $H$ fails to be locally compact is $\mathbb{Q}^{3}$, the group reflexion of $G$.

We claim that the quotient group $G / H$ is not regular. Let $\pi: G \rightarrow G / H$ denote the quotient homomorphism. We can identify $G / H$ with $\mathbb{Q}^{2}$ endowed with a suitable topology.

Let us show that $(0,1) \notin \pi(S)$. Assuming the converse we would find $x \in \mathbb{Q}$ such that $(0,1, x) \in S$. It follows from the definition of $S$ that $x \geq 0$ and there is a sequence $\left(\vec{x}_{i}\right)_{i \in \omega}$ converging to $(0,1, x)$ such that for every $i \in \omega$

$$
\vec{x}_{i}=\sum_{n} \lambda_{i, n} \cdot\left(\frac{1}{n}, 1, z_{n}\right)+\lambda_{i} \cdot(1,0,0)
$$

where all numbers $\lambda_{i}, \lambda_{\text {in }}$ are non-negative and almost all of them vanish. Taking into account that the sequence $\left(\vec{x}_{i}\right)_{i \in \omega}$ converges to $(0,1, x)$ we conclude that

1. $\lambda_{i} \rightarrow 0$ as $i \rightarrow \infty$;

2. $\lambda_{\text {in }} \underset{i \rightarrow \infty}{\longrightarrow} 0$ for every $n$;

3. $\sum_{n} \lambda_{\text {in }}$ tends to 1 as $i \rightarrow \infty$.

To derive a contradiction, we shall prove that $x=\sqrt{2}$. By the choice of the sequence $\left(z_{n}\right)_{n \in \omega}$, for every $\varepsilon>0$ we can find a number $N$ such that $\left|z_{n}-\sqrt{2}\right|<\varepsilon$ for all $n \geq N$. By the conditions (2) and (3), there exists a number $I \in \mathbb{N}$ such that for every $i \geq I$

$$
\max _{n \leq N} \lambda_{\text {in }}<\frac{\varepsilon}{N} \text { and }\left|\sum_{n} \lambda_{\text {in }}-1\right|<\varepsilon .
$$

Then for every $i \geq I$

$$
\begin{aligned}
\left|\sqrt{2}-\sum_{n} \lambda_{i n} z_{n}\right| \leq & \left|\sqrt{2}-\sum_{n} \lambda_{i n} \sqrt{2}\right|+\left|\sum_{n \leq N} \lambda_{i n}\left(\sqrt{2}-z_{n}\right)\right|+\left|\sum_{n>N} \lambda_{i n}\left(\sqrt{2}-z_{n}\right)\right| \leq \\
& \leq \varepsilon \sqrt{2}+\varepsilon+\sum_{n>N} \lambda_{i n} \varepsilon \leq \varepsilon(\sqrt{2}+1+1+\varepsilon) .
\end{aligned}
$$


So $x=\sqrt{2}$ which is impossible. This contradiction shows that $(0,1) \notin \pi(S)$ and thus $\left(0, \frac{1}{n}\right) \notin \pi(S)$ for all $n \in \mathbb{N}$ (since $S$ is a cone).

It remains to prove that for each neighborhood $V \subset \mathbb{Q}^{3}$ of the origin we get $\overline{\pi(V \cap S)} \not \subset$ $\pi(S)$, where the closure is taken in $G / H$. This will follow as soon as we show that $\left(0, \frac{1}{m}\right) \in$ $\overline{\pi(V \cap S)}$ for some $m$. Since $V$ is a (usual) neighborhood of $(0,0,0)$ in $\mathbb{Q}^{3}$, there is $m \in \mathbb{N}$ such that $\frac{1}{m}\left(\frac{1}{n}, 1, z_{n}\right) \in V$ for all $n \in \mathbb{N}$. Then $\frac{1}{m}\left(\frac{1}{n}, 1\right) \in \pi(V \cap S)$ for all $n \in \mathbb{N}$. Observe that the sequence $\left\{\left(\frac{1}{n m}, \frac{1}{m}\right)\right\}_{n \in \mathbb{N}}$ converges to $\left(0, \frac{1}{m}\right)$ in $G / H$ since for each neighborhood $W \subset \mathbb{Q}^{3}$ of $(0,0,0)$ the difference $\left(\frac{1}{n m}, \frac{1}{m}\right)-\left(0, \frac{1}{m}\right)=\left(\frac{1}{n m}, 0\right)$ belongs to $\pi(W \cap S)$ for all sufficiently large $n$. Therefore $\left(0, \frac{1}{m}\right) \in \overline{\pi(V \cap S)} \not \subset \pi(S) \not \supset\left(0, \frac{1}{m}\right)$, which means that $G / H$ is not regular.

A subset $U$ of a topological space $X$ is called canonically open or regular open if $U=\operatorname{int} \bar{U}$. For a topological space $(X, \tau)$, Stone [7] and Katetov [2] considered the topology $\tau_{r}$ on $X$ generated by the base consisting of all canonically open sets of the space $(X, \tau)$. This topology is called the semiregularization of the topology $\tau$. If $(X, \tau)$ is a paratopological group, then $\left(X, \tau_{r}\right)$ is a $T_{3}$ paratopological group [4, Ex. 1.9], [5, p. 31], and [5, p. 28]. (A space $X$ is $T_{3}$ if each closed set $F \subset X$ and every point $x \in X \backslash F$ have disjoint neighborhoods).

In an initial version of the paper [9] Iván Sánchez and Mikhail Tkachenko essentially used the operation of semiregularization in the class of paratopological groups and posed the following

Problem 1. Let $N$ be a closed (not necessarily normal) subgroup of a Hausdorff paratopological group $G$ such that the quotient space $G / N$ is also Hausdorff. Is the semiregularization of the space $G / N$ regular?

Sánchez and Tkachenko remarked that the affirmative answer to the problem would immediately be applied to generalize theorems on productivity of bounded sets in paratopological groups. So we provide this answer here.

Proposition 1. Let $N$ be a subgroup of a paratopological group $G$. Then the semiregularization of the quotient space $G^{\prime}=G / N$ is $T_{3}$.

Proof. Let $U^{\prime}=\operatorname{int} \overline{U^{\prime}} \subset G^{\prime}$ and $x^{\prime} \in U^{\prime}$ be an arbitrary point. Put $U=\pi^{-1}\left(U^{\prime}\right)$ and pick a point $x \in U$ with $\pi(x)=x^{\prime}$. There exists an open neighborhood $V$ of the unit $e \in G$ such that $V x V \subset U$. Then $x^{\prime} \in \pi(x V) \subset \overline{\pi(x V)}=\pi\left(\pi^{-1}(\overline{\pi(x V)})\right)=\pi\left(\overline{\pi^{-1}(\pi(x V))}\right)=\pi(\overline{x V N}) \subset$ $\pi(V \overline{x V N}) \subset \pi(\overline{V x V N}) \subset \pi(\overline{U N})=\pi(\bar{U}) \subset \overline{\pi(U)}=\overline{U^{\prime}}$ (the second equality follows from opennes of the map $\pi)$. Hence $x^{\prime} \in \pi(x V) \subset \overline{\pi(x V)} \subset \pi(V \overline{x V N}) \subset \operatorname{int} \overline{U^{\prime}}=U^{\prime}$.

In a preliminary version of the paper [10], Li-Hong Xie, Piyu Li, and Jin-Ji Tu proved that if a paratopological group $G$ satisfies the separation axiom $T_{i}$ for some $i \in\{1,2,3\}$, then for any compact normal subgroup of $G$, the quotient paratopological group $G / H$ also satisfies the sepatration axiom $T_{i}$. In the following proposition we show that the same inheritance property holds also for $i=0$.

Proposition 2. Let $H$ be a compact normal subgroup of a $T_{0}$ paratopological group $G$. Then the quotient group $G / H$ is $T_{0}$ too.

Proof. Let $\mathcal{B}$ be the family of all open neighborhoods of the unit of the group $G$ and $\mathcal{B}^{\prime}$ be the family of all open neighborhoods of the unit in the quotient group $G / H$. Let $\pi: G \rightarrow$ $G / H$ be the quotient homomorphism. Let $S=\bigcap_{U \in \mathcal{B}} U$ and $S^{\prime}=\bigcap_{U^{\prime} \in \mathcal{B}^{\prime}} U^{\prime}$. Then $S^{\prime} \subset$ 
$\bigcap_{U \in \mathcal{B}} \pi(U H) \subset \pi\left(\bigcap_{U \in \mathcal{B}} U H\right)$. Let $x \in \bigcap_{U \in \mathcal{B}} U H$ be an arbitrary point and $U \in \mathcal{B}$ be an arbitrary neighborhood. There exists a neighborhood $V \in \mathcal{B}$ such that $V^{2} \subset U$. Then $U^{-1} x \supset \overline{V^{-1} x} \supset V^{-1} x$. So $U^{-1} x \cap H \supset \overline{V^{-1} x} \cap H \neq \varnothing($ as $x \in V H)$. Since the set $H$ is compact there exists point $y \in \bigcap_{U \in \mathcal{B}}\left(\overline{U^{-1} x} \cap H\right)=\bigcap_{U \in \mathcal{B}}\left(U^{-1} x \cap H\right)$. So $x \in S y \subset S H$. Hence $S^{\prime} \subset \pi\left(\bigcap_{U \in \mathcal{B}} U H\right) \subset \pi(S H)$ and $S^{\prime} \cap S^{\prime-1} \subset \pi(S H) \cap \pi(S H)^{-1}=\pi\left(S H \cap S^{-1} H\right)$. Let $x \in S H \cap S^{-1} H$ be an arbitrary point. Then there exist elements $s_{1}, s_{2} \in S$ and $h_{1}, h_{2} \in H$ such that $x=s_{1} h_{1}=s_{2}^{-1} h_{2}$. Then $s_{2} s_{1}=h_{2} h_{1}^{-1} \in S \cap H$. But since $H$ is a compact paratopological group, by Lemma 5.4 from [5], $H$ is a topological group. Since $H$ is a $T_{0}$ topological group the space $H$ is $T_{1}$ (in fact, $T_{3 \frac{1}{2}}$ ), so $H \cap S=H \cap \bigcap_{U \in \mathcal{B}} U=\{e\}$. Thus $s_{2} s_{1}=h_{2} h_{1}^{-1}=e$, so $s_{1}=s_{2}^{-1}$ and $h_{2}=h_{1}=h$ for some $h \in H$. Then $x h^{-1}=s_{1}=s_{2}^{-1} \in$ $S \cap S^{-1}=\{e\}$. Hence $x \in H$. Finally, $S^{\prime} \cap S^{\prime-1} \subset \pi\left(S H \cap S^{-1} H\right)=\pi(H)=\{e\}$ and thus the paratopological group $G / H$ satisfies the separation axiom $T_{0}$.

\section{REFERENCES}

1. T. Banakh, A. Ravsky, Oscillator topologies on a paratopological group and related number invariants, Algebraical Structures and their Applications, Kyiv: Inst. Mat. NANU, (2002) 140-153.

2. M. Katětov, On H-closed extensions of topological spaces, Časopis Pěst. Mat. Fys., 72 (1947), 17-32.

3. A. Ravsky, Paratopological groups I, Mat. Stud., 16 (2001), №1, 37-48.

4. A. Ravsky, Paratopological groups II, Mat. Stud., 17 (2002), №1, 93-101.

5. A. Ravsky, The topological and algebraical properties of paratopological groups, Ph.D. Thesis, Lviv University, 2002 (in Ukrainian).

6. A. Ravsky, Pseudocompact paratopological groups, http://arxiv.org/abs/1003.5343

7. M. Stone, Applications of the theory of Boolean rings to general topology, Trans. Amer. Math. Soc., 41 (1937), 375-481.

8. M. Sanchis, M. Tkachenko, Totally Lindelöf and totally $\omega$-narrow paratopological groups, Topology Appl., 155 (2008), №4, 322-334.

9. I. Sánchez, M. Tkachenko, Products of bounded subsets of paratopological groups, Topology Appl., 190 (2015), №1, 42-58.

10. Li-Hong Xie, Piyu Li, Jin-Ji Tu, Notes on (regular) $T_{3}$-reflections in the category of semitopological groups, Topology Appl., 178 (2014), 46-55.

Department of Mathematics, Ivan Franko Lviv National University

Lviv, Ukraine

tbanakh@yahoo.com

Department of Functional Analysis

Pidstryhach Institute for Applied Problems of Mechanics

and Mathematics National Academy of Sciences of Ukraine

Lviv, Ukraine

oravsky@mail.ru 